\title{
Integer Wavelet-based PCA for Face Recognition
}

\author{
Siba Shankar Rout \\ Indian Institute of Technology, \\ Bombay, India
}

\begin{abstract}
Face recognition has received significant attention from researchers in biometrics, pattern recognition and computer vision Communities. Fixed point implementation has limitless advantages than Floating point implementation, i.e. suitable for hardware design, low computational complexity, high speed, less memory required to store, low power consumption \& easy in encoding. Hence fixed point implementation (Integer Wavelet Transform) is best candidate than floating point implementation (Classical Wavelet Transform). There are many different integer wavelet filters which can be used in the transformation stage and the choice of the filter would have some influence on the accuracy rate of the Face Recognition. This paper proposes a PCA on Integer Wavelet domain for face retrieval system which requires less memory as well with less computational complexity than the traditional methods like PCA and Fisher approaches. Aiming at the LL band as feature of image, a feature extraction and image retrieval algorithm using various Integer Wavelet Transform (IWT) is proposed. Since LL subband of wavelet decomposition becomes the input for PCA hence the memory usage can be greatly reduced.
\end{abstract}

All tests and experiments are carried out by using MATLAB as computing environment and programming language. Experimental result shows that the proposed recognition system with very good performance nearly $98 \%$ as recognition accuracy.

\section{General Terms}

Pattern Recognition, Security, Algorithms et. al.

\section{Keywords}

Face Recognition, Integer Wavelet Filters, PCA, Experimental Results.

\section{INTRODUCTION}

Biometric is a very powerful tool to authenticate a person for wide range of applications. One of the applications is face recognition which has received significant attention from researchers in biometrics, pattern recognition and computer vision communities. Because it plays an important role in many real-time application areas such as security measure at Air Ports, Passport Record verification, Criminals list verification in Police Department, identification at front door for Home Security, Visa processing and many more [6]. The face recognition is better biometrics as compared to other biometric traits. The reason behind this as the person's image to be recognized can be captured without the knowledge and co-operation of that person. For any type of biometric recognition, the most important task is to extract distinguishing features from the training biometric traits, which directly dictates the recognition accuracy. Thus, obtaining a significant feature space with respect to the spatial variation in a human face image is very crucial. The images may be represented by their original spatial domain coefficients or by frequency domain coefficients. Features that are not obviously present in one domain may become obvious in the other domain. Unfortunately, Heisenberg Uncertainty theorem implies that the information cannot be compact in both spatial and frequency domain simultaneously. So, neither approach is ideally suited for all kinds of feature distribution. In what follows, we are going to demonstrate the proposed feature extraction algorithm for face recognition, where spatial domain local variation is extracted using wavelet transform [3]. Wavelet has the ability to capture localized time-frequency information of image \& hence motivates its use for feature extraction. The decomposition of the data into different frequency ranges allows us to isolate the frequency components introduced by intrinsic deformations due to expression or extrinsic factors into certain subbands.

Projection-based face recognition has been widely studied during the past two decades [1] [2]. One of the problems using this approach is to require a huge storage space to save the face features obtained from training faces. Keeping in mind Heisenberg Uncertainty Principle and Multi Resolution concept, wavelet can be used for extracting the face feature. This paper proposes a PCA on Integer Wavelet domain for face retrieval system which requires less memory as well with less computational complexity than the traditional methods like PCA and Fisher approaches. Aiming at the LL band as feature of image, a feature extraction and image retrieval algorithm using various Integer Wavelet Transform (IWT) is proposed. Since LL subband of wavelet decomposition becomes the input for PCA the memory usage can be greatly reduced.

\section{PROBLEM STATEMENT}

A general statement of the problem of machine recognition of faces can be formulated as follows (shown in Figure 1)

Given still face images of a subject or person, identify or verify one or more persons from stored database of faces.

\section{PROPOSED WORK}

Wavelet transform has nice features of space-frequency local ization and multi-resolutions. The major reasons for wavelet transforms popularity lie in its complete theoretical framework, the great flexibility for choosing bases and the low computational complexity of $O(\mathrm{~N})$. Classical wavelet transform convert floating point samples to floating point samples because filter coefficients are floating point numbers. Hence even though the input data consists of sequence of integers the resulting transformed samples will be no longer integers. Therefore a mapping would be required to map these floating point numbers to integer numbers. However, in many 

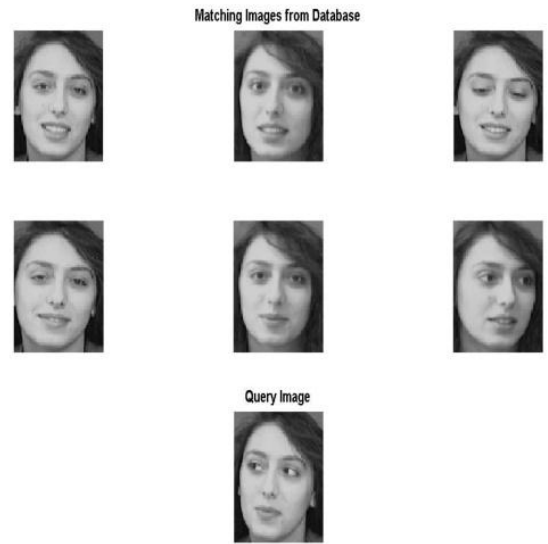

Figure 1: Query Image against Matching Images from Database

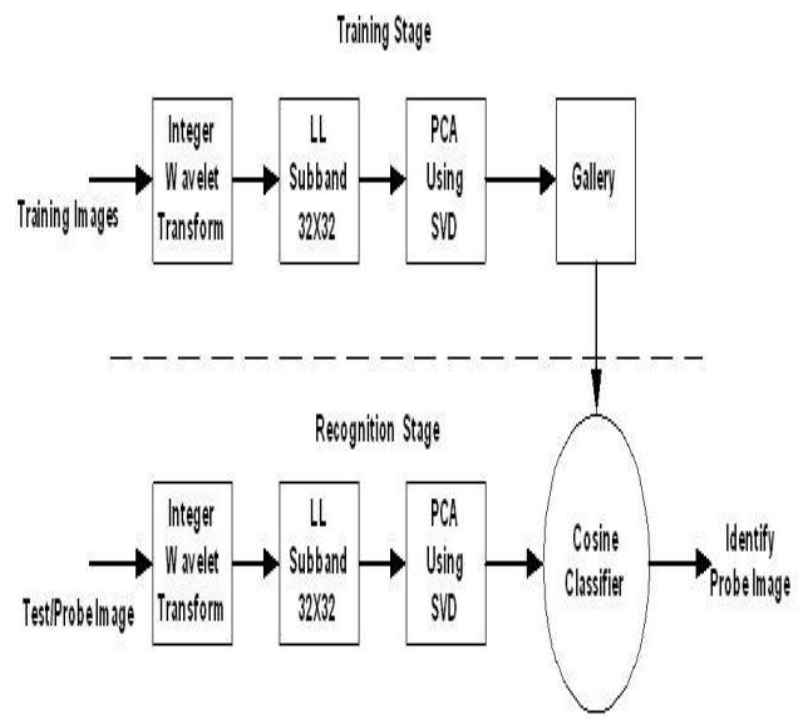

Figure 2: Block diagram of the Proposed Face Recognition System

multimedia applications (like image, video, audio) the input data consists of integer values only. In addition, the storage and the encoding of integer number are easier as compared to floating point numbers [4]. With respect to hardware implementations fixed point implementation uses lower power consumption, compact in size, low computational complexity and fewer transistors than floating point processor. Hence we are interested in various integer wavelet transform that maps integer to integer, while retaining the perfect reconstruction or PR property. Wavelet transform decompose the image on to four parts LL, LH, HL and HH subbands. Out of which LL subband (low frequency components in both vertical and horizontal directions of the original image) contains highestenergy content within the four subbands. It has been proved that LL subband features are insensitive to the facial expressions and also best candidate for facial feature. Wavelet transform is applied to the face image in order to eliminate the effect of different facial expressions [5]. Also, decomposing the face image will reduce the resolution of the image, which in turn, reduces the computation and the memory requirements of the recognition system. The proposed algorithm and its block diagram is shown in Figure 2. There are many different integer wavelet filters which can be used in the transformation stage [4], and the choice of the filter would have some influence on the accuracy rate of the Face Recognition. The experiments are designed to test the effect of the choice of using PCA or not, the choice of wavelet filter, and the depth of decomposition. Hence the problem statement for the proposed face recognition using different wavelet filter can be formulated as follows

Given a list of Wavelet Filters, identify an optimal filter in terms best recognition rate, low memory usage and last not the least with low computational complexity.

The proposed various integer wavelet filters are shown in Figure 3.

\begin{tabular}{|c|c|}
\hline $5 / 3$ & 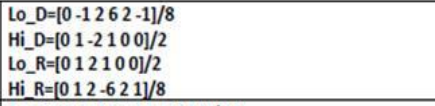 \\
\hline $7 / 5$ & 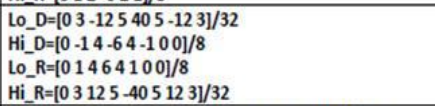 \\
\hline $9 / 71$ & 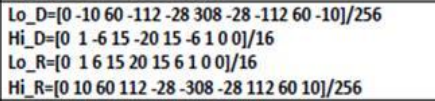 \\
\hline 9/711 & 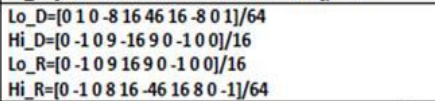 \\
\hline $13 / 7$ & 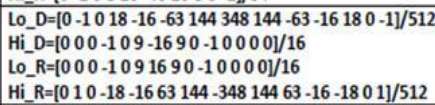 \\
\hline $3 / 5$ & 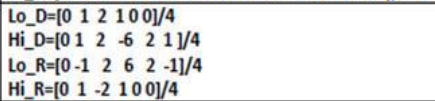 \\
\hline $4 / 4$ & 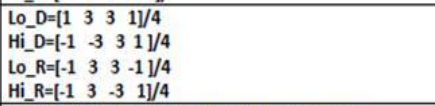 \\
\hline $11 / 5$ & 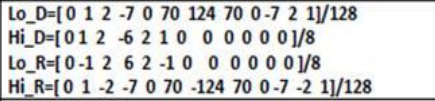 \\
\hline $3 / 1$ & 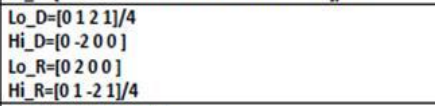 \\
\hline $2 / 6$ & 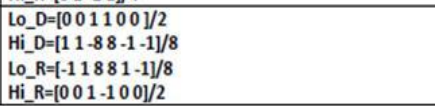 \\
\hline
\end{tabular}

Figure 3: Various Integer Wavelet Filters Used in Face Recognition System

\section{FACE DATABASE}

The proposed algorithm has been tested on three famous face databases for variety of faces for effectiveness of the algorithm. The test results in the different face database produces interesting results from the point of view of recognition success and speed of the face recognition algorithm. In this implementation, all images are resized to a 
uniform dimension of $128 \times 128$ and also normalized to reduce the error due to lighting conditions or various illumination effects.

- FERET: Facial Recognition Technology Database

The FERET Facial Expression Database contains 200 subjects and each subject have 2 facial expressions [8]. 60 person in the FERET database are used in this experiment. For each person, we use first images to form the training set and use the other images to form the testing set. That is, the training images and testing images are 60 and 60 respectively.

\section{- ORL: Olivetti-Att-ORL Database}

The ORL Facial Expression Database contains 40 subjects and each subject have 10 facial expressions [7]. 10 person in the ORL database are used in this experiment. For each person, we use first 6 images to form the training set and use the other 4 images to form the testing set. That is, the training images and testing images are 60 and 40 respectively.

- JEFF: Japanese Female Facial Expression Database

The JEFF Facial Expression Database contains 10 subjects and each subject have 21 facial expressions [9]. 10 person in the JEFF database are used in this experiment. For each person, we use first 6 images to form the training set and use the other 4 images to form the testing set. That is, the training images and testing images are 60 and 40 respectively.

\section{EXPERIMENTAL RESULTS}

All tests and experiments are carried out by using MATLAB as computing environment and programming language. The classifier used is as Cosine Distance Metric. To evaluate the performance of face recognition we used two metric i.e. ."Rank-1 Recognition Accuracy and Recognition Accuracy”. All the experimental result mentioned in Table $1 \&$ Table 2 as well as in Figure $4 \&$ Figure 5. Experimental result shows that the proposed recognition system with very good performance nearly $98 \%$.

\subsection{Rank-1 Recognition Accuracy}

The output of proposed recognition system is a list of sorted reference images in descending order based on similarity with testing (probe) image. It means, the reference image on the top of the list has the highest similarity (lowest distance) with the testing image. If the reference image on the top of the list (top most image) matches with testing (probe) image then we define recognition accuracy as $100 \%$. This is called as"Rank1 Recognition Accuracy" and defined as follows.

\section{$\frac{\text { Number of corrected matching images }}{\text { Total number of testing images }} \times 100 \%$}

The results are depicted in Table $1 \&$ Figure 4.

\subsection{Recognition Accuracy}

The output of proposed recognition system is a list of sorted reference images in descending order based on similarity with testing (probe) image. It means, the reference image on the top of the list has the highest similarity (lowest distance) with the testing image. Assume that there are $N$ images with different expression in database for the testing (probe) image. Hence "Recognition accuracy" with respect to window $N$ is defined as follows.

\section{$\frac{(N u m b e r \text { of correct matching images per subject }) \cap N}{\text { Total number of expression per subject }} \times 100 \%$.}

The results are depicted in Table $2 \&$ Figure 5.

\subsection{Performance of Filters}

In this section we will conclude which integer wavelet filter is the optimal for face recognition rate by observing some of the important factors of the filters.

- In terms of computational point of view $5 / 3$ and $2 / 6$ are truly multiplies less and off course are the best filters.

- 9/7II, 13/7 and 7/5 are the worst filters and should be avoided for feature extraction.

- $5 / 3$ best for all type of images. Because it has compact length, low complexity, highest coding gain and higher recognition rate.

- In terms of lowering dynamic range in transform domain we can say $3 / 1,2 / 6,5 / 3,3 / 5$ and $4 / 4$ are best where as $13 / 7$ is worst among all.

Table 1. Rank-1 or Top Most matching based face recognition using different wavelet filters

\begin{tabular}{|c|c|c|c|}
\hline Filter & $\begin{array}{c}\text { FERET } \\
\text { Database }\end{array}$ & $\begin{array}{c}\text { ORL } \\
\text { Database }\end{array}$ & $\begin{array}{c}\text { JEFF } \\
\text { Database }\end{array}$ \\
\hline $5 / 3$ & $96.66 \%$ & $98.00 \%$ & $95.00 \%$ \\
\hline $7 / 5$ & $95.00 \%$ & $95.00 \%$ & $90.25 \%$ \\
\hline $9 / 71$ & $46.66 \%$ & $57.50 \%$ & $40.00 \%$ \\
\hline $9 / 711$ & $96.66 \%$ & $97.50 \%$ & $92.50 \%$ \\
\hline $13 / 7$ & $96.66 \%$ & $97.50 \%$ & $97.50 \%$ \\
\hline $3 / 5$ & $96.66 \%$ & $97.50 \%$ & $97.50 \%$ \\
\hline $4 / 4$ & $96.66 \%$ & $97.50 \%$ & $97.50 \%$ \\
\hline $11 / 5$ & $96.66 \%$ & $97.50 \%$ & $97.50 \%$ \\
\hline $3 / 1$ & $96.66 \%$ & $97.50 \%$ & $97.50 \%$ \\
\hline $2 / 6$ & $96.66 \%$ & $97.50 \%$ & $97.50 \%$ \\
\hline
\end{tabular}


Table 2. Recognition Accuracy based face recognition using different wavelet filters

\begin{tabular}{|c|c|c|c|}
\hline Filter & $\begin{array}{c}\text { FERET } \\
\text { Database }\end{array}$ & $\begin{array}{c}\text { ORL } \\
\text { Database }\end{array}$ & $\begin{array}{c}\text { JEFF } \\
\text { Database }\end{array}$ \\
\hline $5 / 3$ & $96.66 \%$ & $85.00 \%$ & $84.58 \%$ \\
\hline $7 / 5$ & $95.00 \%$ & $77.50 \%$ & $78.75 \%$ \\
\hline $9 / 71$ & $46.66 \%$ & $25.41 \%$ & $23.75 \%$ \\
\hline $9 / 7 \mathrm{II}$ & $96.66 \%$ & $84.58 \%$ & $83.33 \%$ \\
\hline $13 / 7$ & $96.66 \%$ & $85.00 \%$ & $83.33 \%$ \\
\hline $3 / 5$ & $96.66 \%$ & $85.41 \%$ & $82.91 \%$ \\
\hline $4 / 4$ & $96.66 \%$ & $85.00 \%$ & $83.33 \%$ \\
\hline $11 / 5$ & $96.66 \%$ & $85.00 \%$ & $84.16 \%$ \\
\hline $3 / 1$ & $96.66 \%$ & $85.00 \%$ & $83.75 \%$ \\
\hline $2 / 6$ & $96.66 \%$ & $85.00 \%$ & $82.50 \%$ \\
\hline
\end{tabular}

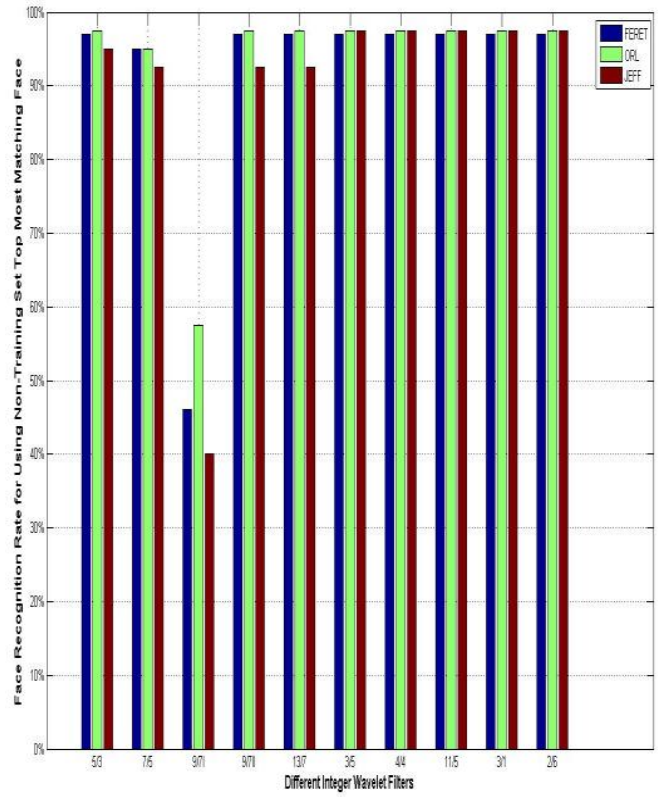

Figure 4: Rank-1 or Top Most matching based face recognition using differ ent wavelet filters

\section{CONCLUSION}

Face Recognition is one of the most popular applications when Biometric Recognition is involved. This paper proposes Integer Wavelet decomposition followed by PCA based projection for extracting the facial feature to create a face recognition system. Experimental result shows that the proposed recognition system with very good performance nearly $98 \%$ as recognition accuracy. Comparing with the methods of applying PCA approach on the original image, this proposed method has the following advantages.
- Low computation as well as low computational complexity in both training and recognition stages since LL subband of wavelet decomposition becomes the input for PCA.

- Suitable for Hardware implementation and design because the proposed algorithm uses integer arithmetic which is based on shift and add operations only.

- Amount of memory required to store floating point arithmetic is very high than integer arithmetic which acquires low memory. Hence integer arithmetic is suitable for resource constrained devices.

- Higher accuracy with speed than traditional methods.

- The Multiresolution representation allows the transmission of the lower resolution version of the image for Face recognition at Server Side which has wide application in Mobile Banking/Transactions.

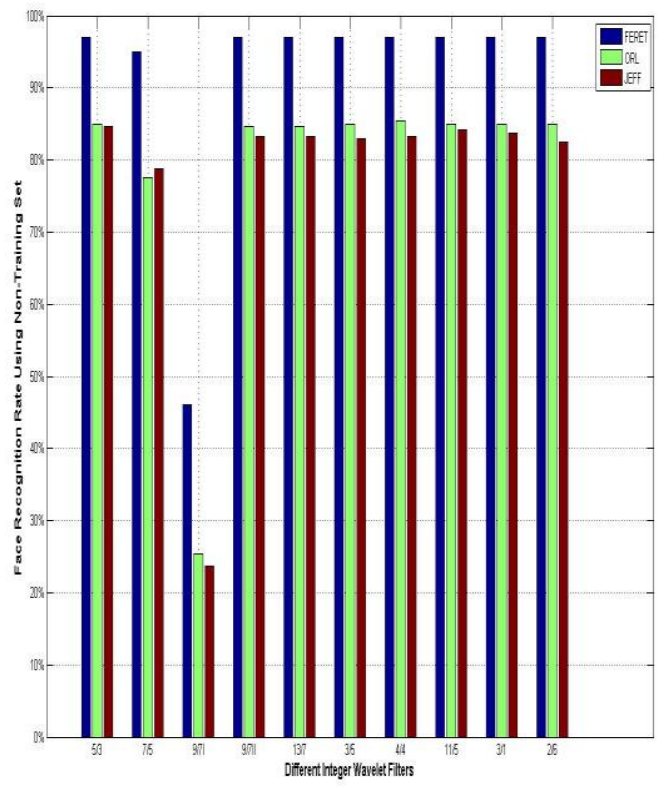

Figure 5: Recognition Accuracy based face recognition using different wavelet filters

\section{REFERENCES}

[1] M. Turk, A. Pentland. Eigen faces for Recognition. Journal of Cognitive Science , 3(1):7186, 1991.

[2] Jon Shlens,"A Tutorial on Principal Component Analysis Derivation, Discussion and Singular Value Decomposition”, 25 March 2003, Version 1.

[3] JEkenel, H. K. and Sanker, B. (2005). Multiresolution face recognition, Image and Vision Computing, Vol. 23, (May 2005) pp. 469-477.

[4] A. R. Calderbank, I. Daubechies, W. Sweldens, and B. L. Yeo, "Wavelet transforms that Map Integers to Integers", it Applied Computational and Harmonic Analysis, VOL. 5, NO. 3, pp 332-369, 1998. 
[5] Jian Huang Lai, Pong C. Yuen and Guo Can Feng, "Face recognition using holistic Fourier invariant features", The Journal of the Pattern Recognition Society, pp 95109, 2001.

[6] Hussein Rady, "Face Recognition using Principle Component Analysis with Different Distance Classifiers", IJCSNS International Journal of Computer Science and Network Security, VOL.11 No.10, (October 2011) pp. 134-144.
[7] AT and T Laboratories, Cambridge, U.K., The ORL Face Database, Available

at: http://www.orl.co.uk/facedatabase.html.

[8] Y P. Phillips, H. Wechsler, J. Huang, and P. Rauss, "The feret database and evaluation procedure for facerecognition algorithms", vol. 16, pp. 295306,April 1998.

[9] The Japanese Female Facial Expression (JAFFE) Database,Available at : http://www.kasrl.org/jaffe.htmlIn Distributed Systems, S. Mullender 